\title{
Molecular Channel Model with Multiple Bit Carrying Molecules
}

\author{
Bige D. Unluturk Ecehan B. Pehlivanoglu Ozgur B. Akan \\ Next-generation Wireless Communications Laboratory (NWCL) \\ Department of Electrical and Electronics Engineering \\ Koc University, 34450, Istanbul, Turkey \\ Email: \{bunluturk, epehlivanoglu, akan\}@ku.edu.tr
}

\begin{abstract}
Molecular communication is a bio-inspired paradigm, proposed to communicate nanomachines via diffusion of molecules through an aqueous medium. The type and structure of the molecules to be propagated bear great importance since they directly affect the modulation structure of molecular communication. We propose a messenger-based molecular communication model where information is encoded on the atoms of polyethylene molecules in the form of $\mathrm{CH}_{3}(\mathrm{CHX})_{n} \mathrm{CH}_{2} \mathrm{~F}$, where $\mathbf{X}$ is either an $\mathbf{H}$ or $\mathbf{F}$ atom, representing 0 and 1 bits, respectively. The encoded polyethylene molecules are released from the transmitter nanomachine, and their propagation towards the receiver is modelled as a Brownian Motion. Using an erasure channel model, our analysis focuses on calculating the capacity of this channel and revealing the parameters affecting it such as molecule size and number of redundant molecules for one transmission.

Index Terms-Molecular communication, communication via diffusion, nanocommunications, nanonetworks, multi-node environment, channel model
\end{abstract}

\section{INTRODUCTION}

Molecular communication is a new bio-inspired paradigm. Biological phenomena, such as inter-cellular communications by the transport of vesicles and hormones enabling inter-organ communications have recently inspired the communications by the dispatch, propagation and reception of molecules in the context of nanomachines. Nanomachines are nanoscale devices, and can be naturally existent (i.e. biological cells) or artificially synthesized (i.e. bioengineered cells) [1]. Molecular communication allows nanomachines to encode outgoing information to molecules, to let messenger molecules propagate in an aqueous medium and to decode information from incoming molecules. Molecular communication is very suitable for nanomachines not only because of its low complexity and low energy requirements but also for its ability to network simple nanomachines to coordinate and carry out complex tasks. Potential application areas of networked nanomachines by molecular communication include but are not limited to medicine, monitoring, quality control and attack detection.

Existing diffusion-based molecular communication models encode information on the concentration of the molecules [1], [2]. In this paper, effects of information encoding on multiple atoms of the messenger molecule on channel capacity are studied. We introduce a messenger-based molecular communication model, in which information is encoded on the atoms of polyethylene molecules which are in the form of $\mathrm{CH}_{3}(\mathrm{CHX})_{n} \mathrm{CH}_{2} \mathrm{~F}$, where $\mathrm{X}$ is either an $\mathrm{H}$ or $\mathrm{F}$ atom, representing a 0 and a 1 bit, respectively. Encoded molecules are assumed to be released into the medium by sender nanomachines and left to propagate following a Brownian Motion process to the receiver nanomachine. Then, the capacity of this channel is derived and the parameters optimizing it are assessed. Novelty of our work can be summarized as follows:

- Multiple bits are assumed to be encoded on a single polyethylene molecule, and are communicated between two nanomachines in a single propagation session.

- Multiple bit encoding on single messenger molecule corresponds to data link layer packets in traditional networking, since it can include the transmitter and receiver identifications and sequence numbers. This fact allows us to move one step forward from physical layer to the data link layer in molecular communications.

- Multiple bit carrying molecules bypass the need for synchronization among nanomachines, by including data packet sequence numbers.

- Results reveal the conditions for which multiple bit carrying molecules is a viable method for molecular communications, for a use case scenario.

The remainder of this paper is organized as follows. In Section II, the molecular communication model with multiple bit carrying molecules is introduced. The channel model and the capacity calculation are given in Section III. The numerical results on the capacity and the effects of the communication channel parameters on it are provided in Section IV. Finally, a brief conclusion on the results are given in Section V.

\section{Molecular Communication Model}

The messenger molecule considered for this model is partially fluorinated polyethylene, i.e., $\mathrm{CH}_{3}(\mathrm{CHX})_{n} \mathrm{CH}_{2} \mathrm{~F}$, carrying $n$ bits of information via diffusion in the communication medium. Some forms of these fluorinated polythylene molecules are utilized as oxygen carriers in artificial blood formulations [3]. In our model, we focus on these molecules called fluorinated hydrocarbon perfusants and assume that they propagate in human blood as a use case.

The value of $n$ in the molecule's formula can reach up to $10^{9}$ bits [4], making large chunks of information transferable. However, because of the slow diffusion of large molecules, 
propagation delays definitely rise. Thus, it is important to find an optimum value for molecule size in the capacity sense.

Our model embodies five main processes; namely; information encoding process, transmission process, propagation process, reception process and information decoding process [5].

\section{A. Information Encoding Process}

In this model, the transmitter (TX) nanomachine generates messenger molecules carrying $n$ bits of information. Firstly, an ideal messenger molecule should be easily recognized by nano receptors due to the special structure of these molecules containing a distinctive head and tail. Secondly, these molecules should be bioinactive, not easily corrupted by natural processes before they reach the receiver (RX) nanomachine [4].

Partially fluorinated polyethylene molecules are candidates for such messenger molecules as suggested in [6]. A partially fluorinated polyethylene molecule is in the form $\mathrm{CH}_{3}(\mathrm{CHX})_{n} \mathrm{CH}_{2} \mathrm{~F}$, where $\mathrm{X}$ is either an $\mathrm{H}$ or $\mathrm{F}$ atom, representing the 0 and 1 bits, respectively. $\mathrm{CH}_{3}$ and $\mathrm{CH}_{2} \mathrm{~F}$ form the distinctive head and tail of the molecule, providing a decoding order for the receiver. Hence, headers that are assured to be read first, may be added to the front of the data which may be used to store TX and RX identifiers, parity and checksum bits, flags that are needed to step up from the physical layer to data link layer. This structure enables the knowledge transfer from classical wireless communications techniques to molecular communication for some concepts such as routing, medium access control, reliability.

Furthermore, the carbon chain of polyethylene is assumed to contain the encoded atoms only in one side of the chain for the sake of readibility and the other side is assumed to be full of $H$ atoms. We assume also that an encoded polyethylene carries $50 \% H$ and $50 \% F$ atoms on the average on the coded side of the molecule to have equal diffusion coefficients for each encoded molecule. According to these assumptions, the information density of this molecule is $d_{\text {message }} \sim 26$ bits $/ \mathrm{nm}^{3}$, a significantly higher number compared to a more common molecule, DNA, whose information density is $\sim 1 \mathrm{bit} / \mathrm{nm}^{3}$ [4] which leads us to prefer fluorinated polyethylene to DNA.

The information capacity of a single molecule increases with increasing $n$, in exchange for a larger molecule size which slows down the diffusion of the molecule. The diffusion coefficient for spherical particles through liquid is expressed by the Einstein-Stokes equation [7] as $D=k_{B} T / 6 \pi \eta r$, where $k_{B}$ is the Boltzmann's constant, $T$ is the absolute temperature, $\eta$ is viscosity and $r$ is the radius of the nanomachine [7].

Molecule Shift Keying (MoSK), a molecular modulation technique introduced in [8], can be exploited for the information encoding process of this study. This modulation scheme requires $2^{n}$ different molecules to represent $n$ bits of logical information. For the transmission of an intended symbol, one of these molecules is released by the transmitter and the receiver decodes the intended symbol according to the type of the received molecule. An example $n=2$ bit constellation diagram for Quadruple MoSK is shown in Fig. 1 [8]. In our

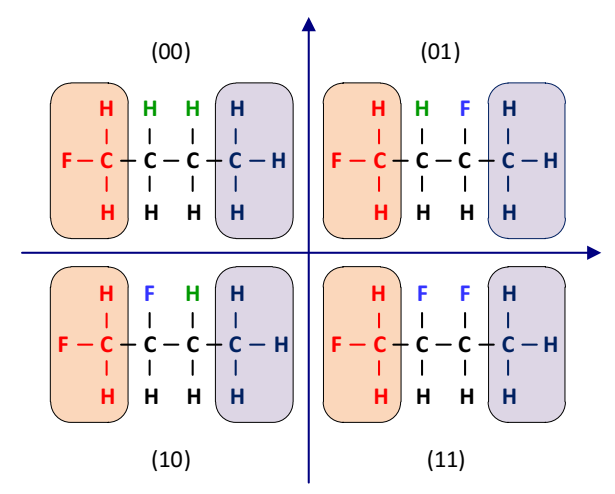

Fig. 1. Constellation diagram for QMoSK modulation, $n=2$ [8].

case, $n$ can reach up to the order of $10^{4}$ and corresponding constellation diagrams are similar to the diagram in Fig. 1.

\section{B. Transmission Process}

The TX nanomachine is assumed to have a spherical shape and the messenger molecules are released to the medium from the boundary of the nanomachine. This emission process is uniformly distributed over this spherical boundary, called the emission boundary as seen in Fig. 2. Since the messenger molecules are assumed to propagate by Brownian Motion in the medium in all directions, the probability that a single molecule reaches the receiver in a given time is very small. Besides, the messenger molecules can be degraded by the environment. Hence, for the sake of reliability, the transmitter emits redundant copies of the molecule, namely $N_{M}$ copies, for each message in order to raise the reception probability. The booster effect on channel capacity of using extra molecules where the information is encoded in the concentration of the molecules is illustrated in [1]. In this study, we use the same method to boost the channel capacity where the information is encoded in the type of the molecule.

Emission of the molecules takes place instantaneously which is inspired from the emission of pheromones in animals in alarming situations such as threats from predators, injured conspecifics, and exposure to toxic compounds [9].

\section{Propagation Process}

Messenger molecules released from the transmitter propagate in the fluid medium by diffusion. The random motion of the messenger molecules is modelled as Brownian. We assume that the total concentration of emitted molecules are

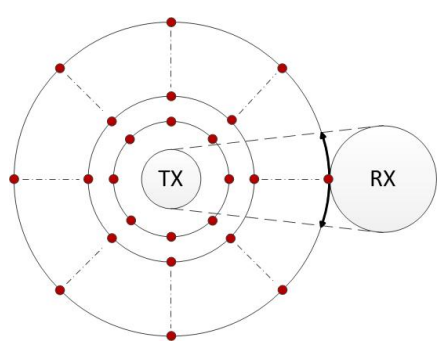

Fig. 2. Particle propagation and detection processes. 
much lower than the concentration of all fluid molecules. Therefore, the interactions between molecules are neglected and the movement of each molecule is assumed independent. Considering these assumptions, we can express the concentration of the molecules by Fick's law of diffusion [2]. For instantaneous emission of the messenger molecules described in Section II-B, the first hitting time $t$ of an emitted molecule to a spherical surface at a distance $d$ from the emission boundary is distributed as an inverse Gaussian pdf [1], i.e.,

$$
f(t)=\frac{d}{\sqrt{4 \pi D t^{3}}} \exp \left(\frac{-d^{2}}{4 D t}\right), \quad t \geq 0,
$$

where $D$ is the diffusion coefficient.

\section{Reception Process}

RX nanomachine has specific receptors which bind to the messenger molecules arriving to their vicinity. When a messenger molecule hits the boundary of the receiver, it is assumed to be instantly captured for decoding.

We assume that reception of only one of the $N_{M}$ molecules released for a single message is sufficient for decoding the message. Thus, we need a mechanism to recognize the extra molecules arriving to the receiver after the reception of the first molecule carrying the same information. Besides, residual molecules from the previous messages may arrive to the receiver during the reception of the current message and may be misinterpreted as the current message. To cope with these two problems, the transmitter adds extra message identifier bits to the regular information bits such that the receiver can identify to which message that the incoming molecule belongs and does not decode the molecules arriving to the receiver after the reception of the first molecule with the same identifier.

\section{E. Decoding Process}

By exploiting the special structure of the molecule described in Section II-B, the receiver reads the information encoded on the incoming molecules, right after reception. The head and tail structure enables the receiver to know from which end of the molecule it should start decoding. Furthermore, since only one side of the molecule is encoded, the receiver also knows which side should be decoded. The molecule captured by the receiver is decoded by the demodulation of the incoming molecule generated according to MoSK presented in Section II-B. An alternative decoding scheme is reading the molecule bit by bit. The difference in interaction energies between the coded $H$ and $F$ atoms and the boron atom, $B$, of a $C_{5} H_{5} B$ or $C_{3} H_{3} N_{2} B$ probe reveals the type of the coded atom [10].

\section{CHANNEL MODEL}

Using the communication model proposed in Section II, we put forward a channel model between a TX and a RX nanomachine for the messenger based molecular communication.

The message-carrying fluorinated polyethylene molecules are generally biocompatible, even though a thorough study for toxicity levels of different types of polyethylene molecules is still required [4]. Some of the fluorinated polyethylene

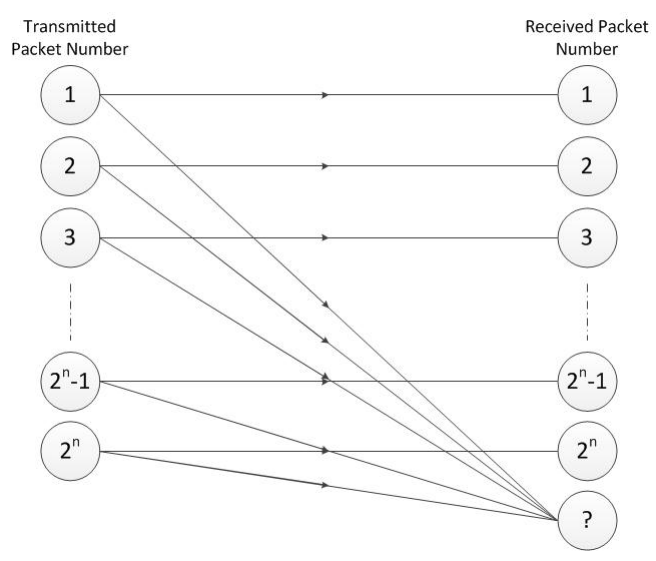

Fig. 3. Block erasure channel.

molecules are FDA approved to be used in artificial blood formulations [3]. Thus we consider that the proposed communication model operates in human body transferring information by polyethylene molecules propagating in blood.

In this model, transmitter emits multiple molecules of each message for reliability. When one of the emitted molecules reaches the receiver, information transmission is considered to be successful. Otherwise, when none reach the receiver, the transmission is considered erroneous. Although polyethylene molecules are considered stable and chemically inert [3], they are cleared from blood by the immune system. Monocytes or macrophages ingest these molecules and clear them rapidly from the blood [11]. For successful reception of a messenger molecule, it has to reach the RX nanomachine before being caught by the immune system. The environment does not damage the bit information carried on $H$ and $F$ atoms since the fluorinated polyethylene molecules are proven to be chemically inert [3]. If one molecule reaches the receiver, it completely yields the message without any bit error. Otherwise, when none of the messenger molecule copies arrive to the receiver due to random motion or phagocytosis, information carried by these messenger molecules is assumed to be erased. In this case, the communication is modelled by a block erasure channel, illustrated in Fig. 3, where each block of $n$ bits are either jointly received with probability $(1-\alpha)$ or jointly erased with probability $\alpha$. The information sent over the molecular channel is $X^{n} \in\{0,1\}^{n}$ and the information received is $Y^{n} \in\{0,1\}^{n}$ if the block is not erased in the channel.

This channel model does not require synchronisation, since the messenger molecules carry not only data bits but also identification bits. From the sequence numbers, erased messages by the channel can be recognized by the RX nanomachine.

To find the probability of erasure $\alpha$, it is assumed that messenger molecules are cleared from blood at an exponential rate $\lambda$ [3]. Then, a released polyethylene molecule has a lifetime $\tau=1 / \lambda$, after which it is considered to be cleared from blood. $q$, probability that a molecule reaches the receiver 
in $\tau$ seconds, before lifetime of the molecule, is given by

$$
q=\int_{0}^{\tau} f(t) d t
$$

where $f(t)$ is the probability density function of first hitting time of a molecule to the receiver, given in (1). Then, the probability of erasure, $\alpha$ which is the probability that none of the $N_{M}$ molecules belonging to the same message arrive to the receiver is expressed by

$$
\alpha=(1-q)^{N_{M}} .
$$

Knowing the block erasure probability $\alpha$, the capacity of this channel can be calculated as

$$
C_{e}=(1-\alpha) n .
$$

By inserting (2) and (3) into (4), the capacity expression is

$$
C_{e}=\left(1-\left(1-\int_{0}^{\tau} f(t) d t\right)^{N_{M}}\right) n \text { bits/channel use. }
$$

Clearly, capacity of the molecular channel depends on the lifetime of the polyethylene molecule in the blood $\tau$, the number of redundant molecules $N_{M}$, the diffusion coefficient $D$, the distance between the transmitter and the receiver $d$ and the number of bits encoded onto a molecule $n$.

While the effects of $N_{M}, \tau$ and $d$ on the erasure channel capacity is straightforward, the effect of a change in $n$ is not clear. Increasing $n$ seems to allow higher capacity when $D$, the diffusion coefficient of the molecules is fixed. However, when $n$ increases, it means that more atoms are added in the polyethylene molecule which makes it larger. The larger molecule size decreases the diffusion coefficient, $D$, thus the diffusion speed of the molecules. Hence, the number of molecules able to reach the receiver before $\tau$ lowers which have a negative influence on the capacity. Therefore, it is handy to find $n$ which maximizes the capacity for the erasure channel.

\section{NUMERICAL RESUlTS}

In this section, we assess the effects of numerous communication parameters on molecular erasure channel capacity.

\section{A. Effect of Molecule Size}

Here, we consider the effect of varying $n$ on the polyethylene molecule formula $\mathrm{CH}_{3}(\mathrm{CHX})_{n} \mathrm{CH}_{2} \mathrm{~F}$. The effect of $n$ on the successful reception of the molecule within its $\tau$ lifetime is not trivial. Fig. 4 assesses the achievable erasure channel capacity for varying $n$. For this assessment, the lifetime $\tau$ is fixed to 100 seconds which is an approximate value for the lifetime of fluorinated polyethylene in the human intravascular system for artificial blood applications [11], and TX-RX distance is assumed $50 \mu m$ [4]. Human blood has a viscosity in the range $20 \times 10^{-3}$ to $40 \times 10^{-3} \mathrm{~kg} / \mathrm{m}-\mathrm{s}$ [4].

In Fig. 4, erasure channel capacities for different medium viscosity levels are presented. Clearly, the viscosity of the medium has a great impact on the optimal alphabet size for capacity. As expected, with increasing viscosity, optimal $n$ values that achieve maximum channel capacity decrease.

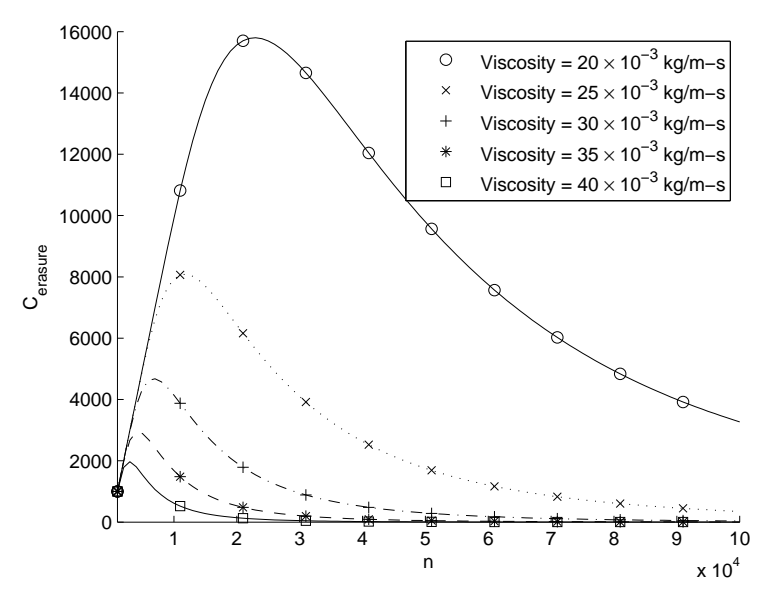

Fig. 4. Capacity of molecular erasure channel for varying $n$ (i.e., molecular size) with different viscosities

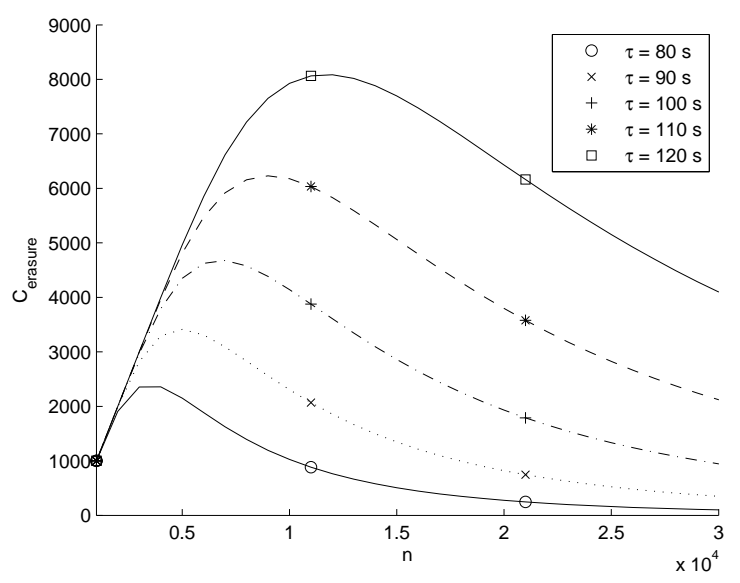

Fig. 5. Capacity of molecular erasure channel for varying $n$ (i.e., molecular size) with different lifetimes, $\tau$

\section{B. Effect of Lifetime $\tau$}

Since the messenger molecules move randomly in the propagation medium, not all the $N_{M}$ molecules emitted for a message reach the receiver. To improve the probability of reception, synthesizing molecules with longer lifetime against phagocytes is considered. In Fig. 5, the capacity of the proposed molecular channel is evaluated with respect to varying $n$ for different lifetimes. The viscosity of the medium is fixed to $30 \times 10^{-3} \mathrm{~kg} / \mathrm{m}-\mathrm{s}$ and the number of emitted molecules is fixed to 1000. As seen Fig. 5, an increase in $\tau$ significantly improves the capacity. Thus, to find the optimal $n$, the lifetime $\tau$ should also be considered as a significant parameter.

\section{Effect of Number of Redundant Molecules $N_{M}$}

Because of the random motion of messenger molecules, successful reception cannot be guaranteed. To increase the probability of reception, redundant identical molecules are used. $N_{M}$ identical copies of the messenger molecule are released. In Fig. 6, it is clearly seen that as the number of redundant molecules is higher, the capacity becomes higher since the probability of reception of the message is increased. 


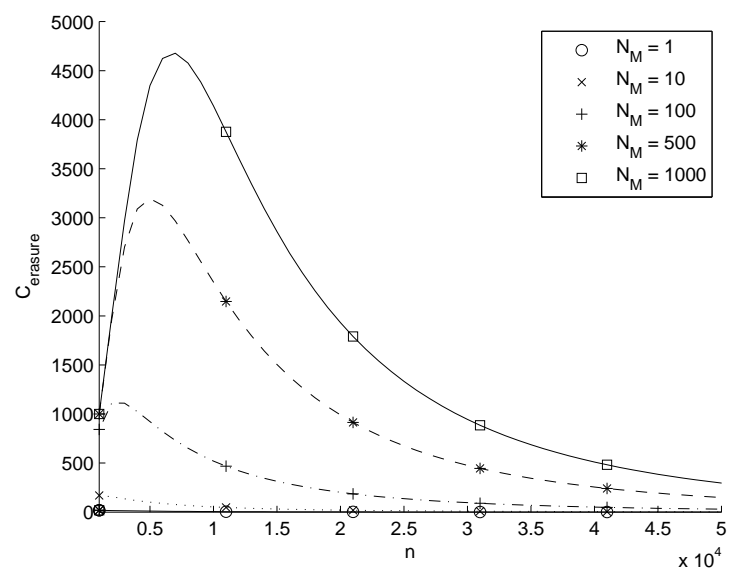

Fig. 6. Capacity of molecular erasure channel for varying $n$ (i.e., molecular size) with different number of redundant molecules, $N_{M}$

\section{Optimal Number of Bits In a Molecule $n$}

For the fluorinated polyethylene, Fig. 4,5 and 6 illustrate that the number of bits encoded on a molecule, $n$, which maximizes the capacity, changes with changing molecule lifetime, $\tau$ and number of redundant molecules, $N_{M}$. An analytical solution for the optimal $n$ using the capacity expression (4) can not be derived which led us to obtain a closed-form expression using a numerical approach by means of MATLAB Optimization Toolbox and Surface Fitting Toolbox.

Firstly, optimal number of bits $n$ is calculated and plotted in $3 \mathrm{D}$ with respect to the variation of two important parameters of optimization problem, namely, $\tau$ and $N_{M}$. Then, a surface is fitted on these data points as in Fig. 7. According to the results of surface fitting, a closed-form expression in the form of a polynomial of the third degree for each parameter is derived.

In Fig. 7, the optimal number of bits calculated numerically shown by the points and the surface fitted on them are illustrated. Using the parameters of the fitted surface an expression for optimal $n$ is found as

$$
\begin{aligned}
& n_{\text {opt }}=26.55-1.163 N_{M}+8.532 \tau+0.0023 N_{M}^{2} \\
& \quad+0.012 N_{M} \tau-0.3019 \tau^{2}-8.758 \times 10^{-7} N_{M}^{3} \\
& \quad+4.362 \times 10^{-5} N_{M}^{2} \tau-0.00028 N_{M} \tau^{2}+0.0024 \tau^{3}
\end{aligned}
$$

Besides finding the optimal number of bits encoded in a molecule in terms of the lifetime of the molecule $\tau$ and the number of redundant molecules $N_{M}$, we should also assess the feasibility of the described communication system. As the messenger molecules suggested in this study should operate in human blood, they should be able to tolerate the body temperature. For example, fluorocarbons with $n<5$ have boiling points below $37^{\circ} \mathrm{C}$. During the phagoctosis of the messenger molecules by the immune system agents, molecules with $n$ larger than $10^{6}$ causes loss of phagocytic functions of these agents [4]. This phenomena settle upper and lower bounds for $n$. Considering Fig. 5 and 6, when the viscosity of the medium is set to the whole blood viscosity, the optimal

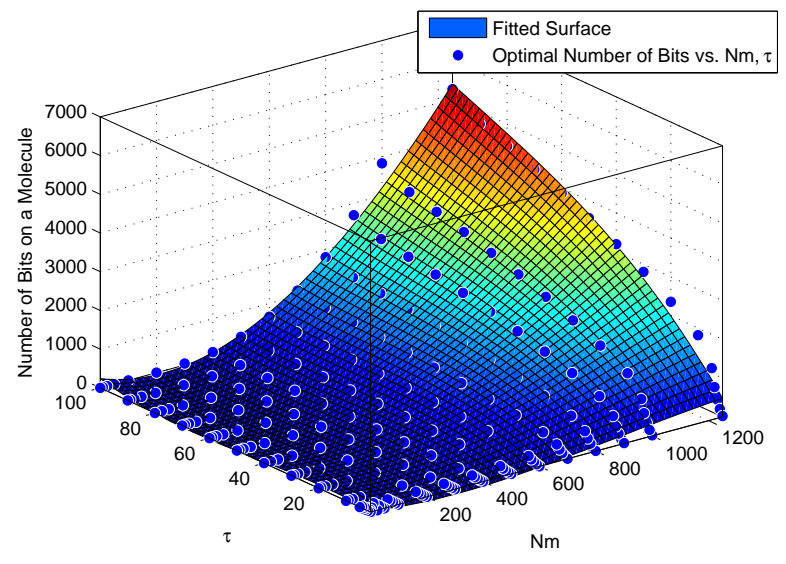

Fig. 7. The fitted surface for the optimal number of bits, $n$

number of bits lies between $0.25 \times 10^{4}$ and $1 \times 10^{4}$. These values satisfies the feasibility conditions stated previously.

\section{CONCLUSION}

In this study, a molecular communication scheme with multiple bit carrying fluorinated polyethylene messenger molecules is presented. The communication channel with Brownian propagation is modelled as a block erasure channel and its capacity expression is derived. Numerical results reveal that when parameters such as the number of redundant molecules and the slot length are properly tuned, very high capacities are achievable. Optimal number of bits in capacity sense is approximated as a function of these parameters. Achievable capacity and the polyethylene biocompatibility makes the proposed model a candidate for molecular communication systems for nature and human body.

\section{REFERENCES}

[1] T. Nakano, Y. Okaie, and J. Q. Liu, "Channel Model and Capacity Analysis of Molecular Communication with Brownian Motion," IEEE Communications Letters, vol. 16, no. 6, pp 797-800, 2012.

[2] L. Parcerisa , I.F. Akyildiz, "Molecular Communication Options for Long Range Nanonetworks", Computer Networks Journal (Elsevier), vol. 53, no. 16, pp. 2753-2766, 2009.

[3] T. F. Zuck, J. G. Riess, "Current Status of Injectable Oxygen Carriers, Crit. Rev. Clin. Lab. Sci. no. 31, pp. 295-324, 1994.

[4] R.A. Freitas, Nanomedicine, Volume I: Basic Capabilities, Landes Bioscience, Georgetown, TX, 1999.

[5] B. D. Unluturk, D. Malak, O. B. Akan, "Rate-Delay Tradeoff With Network Coding in Molecular Nanonetworks," IEEE Transactions on Nanotechnology, vol. 12, no. 2, pp. 120-128, 2013.

[6] K. E Drexler, Nanosystems: Molecular Machinery, Manufacturing, and Computation, John Wiley \& Sons, NY, 1992.

[7] S. Lindsay, Introduction to Nanoscience, Oxford University Press, Oxford, UK, 2009.

[8] M. S. Kuran, H. B. Yilmaz, T. Tugcu, I. F. Akyildiz, "Modulation Techniques for Communication via Diffusion in Nanonetworks" in IEEE International Conference on Communications, Kyoto, Japan, Jun. 2011, pp. 1-5.

[9] T. D. Wyatt, Pheromones and animal behaviour: communication by smell and taste, New York: Cambridge University Press, 2003, pp. 9-10.

[10] C. W. Bauschlicher Jr., A. Ricca, R. Merkle, "Chemical storage of data," Nanotechnology, vol. 8, no. 1, pp. 1-5, 1997.

[11] Winslow, R. M. (2006), Current status of oxygen carriers (blood substitutes): 2006. Vox Sanguinis, 91: 102110. 